\title{
Servicios ambientales del bosque: ensayo en una cuenca atlántica europea con base en la experiencia de Centroamérica
}

\author{
Oreina Orrantia Albizu'1, M. Mercedes Ortega Hidalgo², Olman Quirós Madrigal ${ }^{3}$ \\ $\&$ Javier Loidi Arregui ${ }^{4}$ \\ 1. CIMAS, Innovación y Medio Ambiente S.L. Área de Biodiversidad y Territorio, Avda. Madariaga 1 - 3, 48014 Bilbao, \\ España; oreina.orrantia@cimasconsultores.com \\ 2. Departamento de Genética, Antropología, Física y Fisiología animal, Facultad de Ciencia y Tecnología, Universidad \\ del País Vasco, Apdo. 664, 48080 Bilbao, España; mercedes.ortega@ehu.es \\ 3. Escuela de Economía Agrícola, Universidad de Costa Rica, 2060 San José, Costa Rica; olmanq@agro.ucr.ac.cr \\ 4. Departamento de Biología Vegetal y Ecología, Facultad de Ciencia y Tecnología, Universidad del País Vasco, Apdo. \\ 664, 48080 Bilbao, España; javier.loidi@ehu.es
}

Recibido 05-VII-2007. Corregido 16-VI-2008. Aceptado 16-VII-2008.

\begin{abstract}
Environmental services of the forest: an essay in an European Atlantic river basin based on a Central American experience. A Conservation Interest Index (CI) was designed to numerically assess the natural quality or value of a given terrestrial area. This CI has been applied along the Golako River Watershed (Biosphere Reserve, Basque Country, Spain). The area, although benefiting from some protection, is strongly influenced by human activities (forestry and cattle breeding). The CI is based on both available cartographic information about vegetation and fieldwork, the later needed to provide estimations for the various descriptors included in this index: in this way, a particular vegetation fragment received a final score on a scale from 0 to 1 000. A set of 9 Vegetation Units has been defined to analyze the current vegetation profile and, a ten plot ( $500 \mathrm{x}$ $500 \mathrm{~m}$ ) uniformly distributed sampling design has been implemented. Landscape homogenization is high where main land use relies on timbering, contrasting with the more heterogeneous and fragmented profile related to rural activities. At a height of more than $150 \mathrm{~m}$ forest appears to be dominant while human occupation becomes patchy, whereas abruptness restraints farms to locations below $100 \mathrm{~m}$. Concerning the index performance, gradual differences have been displayed by the forest, which appears as the only vegetation unit attaining values above 500 (50\% in the index scale), mature forest ranking highest (860), followed closely by the riparian forest. We have developed a formula to translate environmental value into economic benefit to promote conservation work at private property level, imitating the initial work undergone in Central American countries, where environmental services are rewarded irrespective of their conservation status. Rev. Biol. Trop. 56 (4): 2087-2098. Epub 2008 December 12.
\end{abstract}

Key words: Environmental services, quantitative vegetation assessment, conservation interest index, Basque Country, Reserva de la biosfera de Urdaibai.

El Pago por servicios ambientales (PSA) es una herramienta para promover la conservación de los recursos naturales de uso en todo el mundo (Vinicio 1998, FAO 2003, FONAFIFO 2003). Este esquema de financiación del mantenimiento de la cobertura arbórea se viene utilizando en Costa Rica desde 1997, donde la entrada de divisas debidas al ecoturismo supone un aporte importante a la economía. La estrategia de PSA nació como un mecanismo para frenar la deforestación y la degradación ambiental que venía sufriendo el país desde la década de los sesenta (Ortiz et al. 2003).

En Costa Rica, dicho esquema es utilizado para atender tres unidades de paisaje: las plantaciones, los bosques y los sistemas agroforestales, en los que se incluyen usos de la tierra para agricultura, ganadería y plantación forestal.

Los beneficios reconocidos de estas tres unidades son el papel que juegan en la 
protección del recurso hídrico, la conservación de la biodiversidad, la belleza escénica y la retención de carbono (mitigación de emisiones de gases de efecto invernadero). Así mismo, la financiación de este esquema se encuentra en el impuesto selectivo a hidrocarburos y combustibles, la asunción de costos ambientales en tarifas de servicios públicos, alianzas estratégicas entre FONAFIFO (Fondo Nacional de Financiamiento Forestal) y empresas privadas y el Fondo de Carbono Internacional (Certified Tradable Offsets, tramitados primeramente por la Oficina Costarricense de Implementación Conjunta). Su gestión es llevada a cabo por la oficina técnica Fondo Nacional de Financiamiento Forestal (FONAFIFO 2003).

El planteamiento realizado para aplicar esta herramienta en la Península Ibérica ha sido adaptar los conocimientos costarricenses a la situación y necesidades de la zona que, en el caso del área de estudio escogida (franja atlántica), se caracteriza por un paisaje montañoso, con abundantes núcleos de población dispersa que determinan en su conjunto elevadas densidades de población. En nuestros días, se hace evidente un aumento creciente de las áreas urbanizadas que es paralelo al deterioro de los espacios naturales, a la vez que crece el reconocimiento científico y social de los valores del bosque y el convencimiento de la necesidad de conservarlo por su contribución en el mantenimiento de la Sociedad del Bienestar. En este contexto, y siguiendo las recomendaciones de expertos (Meaza y Cadiñanos 2000), hemos estimado de utilidad abordar, en primer lugar, la evaluación del grado de calidad natural de los distintos territorios mediante criterios científicos, con un método repetible y que proporcione resultados numéricos, de modo que se pueda medir la contribución de las áreas naturales razonablemente bien conservadas a la preservación de la calidad de vida de las poblaciones humanas. Estas valoraciones representan el paso previo para otorgar primas monetarias cuyo objetivo final persigue la conservación del territorio natural.

Dicha actuación se enmarca en el ámbito legal en la Estrategia Ambiental vasca de
Desarrollo Sostenible, EADS (2002-2020) (Gobierno Vasco 2002). El Programa Marco Ambiental (PMA) que desarrolla esta estrategia sigue las directrices de la "Estrategia de la Unión Europea para un desarrollo sostenible" y las cinco Metas Ambientales definidas en la EADS. En su primera fase (PMA 2000-2006) establece varios objetivos y compromisos a asumir que encajan con la línea seguida en este trabajo.

Entre los compromisos de la Meta 1 "Garantizar un aire, agua y suelos limpios y saludables", se citan: "Mantener o aumentar la superficie forestal por los beneficios que de la misma se derivan tanto para la depuración del aire, régimen hidrológico de las aguas y control de la erosión" y "Aumentar la superficie con compromisos agroambientales hasta las 55000 ha".

Así mismo, entre los compromisos a largo plazo 2007-2020 de la Meta 3, se encuentran: “(...) establecer un régimen normativo de primas compensatorias para bosques autóctonos sin rentabilidad económica directa o actividad económica en un plazo superior a los 100 años, en interés a su función social y ecológica", "Aumentar (...) la superficie de bosque" y "Alcanzar un aprovechamiento extensivo del suelo agrícola (...)".

Para "Limitar la influencia del cambio climático", Meta 3 de la Estrategia, el PMA fija como objetivo "Aumentar los sumideros de carbono", concretándolo en una "Promoción de usos imperecederos de la madera".

Las metas anteriormente propuestas recogen, en lo esencial, la valoración de las funciones que el bosque natural presta a las sociedades humanas (ver Campbell y Doeg 1989, Maridet et al. 1996, Edeso et al. 1999, Elosegi et al. 1995, Montori et al. 2001, Grieve 2003 a, Yeakley et al. 2003, Scherr et al. 2006) entre las cuales los especialistas destacan:

- Biodiversidad: genética, específica y de los hábitats

- Edafogenética: génesis y mantenimiento de la calidad y profundidad de los suelos

- Mitigación de la erosión 
- Regulación del régimen hídrico: atenuación de las avenidas

- Depuración de aguas en casos de contaminación orgánica

- Reservorio de carbono

Por consiguiente, la herramienta de valoración utilizada en este trabajo, ha abordado la cuantificación de estas funciones en términos de un índice global de conservación (IC) que determinaría el grado de calidad ambiental de un área geográfica determinada. Así mismo, el trabajo formula una primera aproximación a la conversión del índice de calidad ambiental en primas monetarias, adaptado a una estructura de propiedad del suelo privada, basada en el pequeño propietario.

\section{MATERIALES Y MÉTODOS}

La investigación se ubicó en la Reserva de la biosfera de Urdaibai (Golfo de Vizcaya, Norte de España, UTM 30TWP2887 a 30TWP3198), concretamente en la cuenca hidrográfica del río Golako. Se eligieron 10 estaciones de estudio de 500 x $500 \mathrm{~m}$ ( 25 ha/estación) distribuidas uniformemente a lo largo de la cuenca del Golako, con la finalidad de conseguir una muestra equilibrada de los ecosistemas existentes (Loidi 2005), llamados Unidades de paisaje (UP) utilizando, así mismo, información fitosociológica (Loidi et al. 1997, Rivas-Martínez et al. 2001) y mapas de vegetación potencial (Gobierno Vasco 1990).

Cada estación se situó junto a dicho arroyo, al objeto de dotar de un nexo de unión al conjunto de las estaciones. Las zonas escogidas no estaban canalizadas, presentaban bordes naturales y un acceso relativamente cómodo desde el valle, a través de pistas forestales, carreteras o senderos. Las estaciones se seleccionaron de manera que se abarcara toda la cuenca y sus diversos ecosistemas. Se dispersaron las estaciones evitando una distribución aleatoria por cuanto se quería recoger la totalidad de situaciones en los diferentes ámbitos de la cuenca. Por ello, hubo estaciones tanto en la vega como a media ladera, y tanto cercanas a núcleos rurales como alejadas de éstos.
Se procedió a recorrer a pie las 250 hectáreas que suman las superficies de todas las parcelas de muestreo, reconociendo en el lugar las diferentes UP y manchas de cada unidad existentes. Tras una primera aproximación se procedió a trabajar en mapas informáticos topográficos, de vegetación y ortofotos de diferentes años, así como con una base de 350 fotografías digitales tomadas durante el trabajo de campo. Esta aproximación permitió definir nueve unidades de paisaje (Cuadro 1) presentes en el conjunto de las estaciones y utilizarlas para concretar la vegetación de las manchas existentes en cada estación. El estudio de campo se realizó entre septiembre de 2003 y mayo de 2004 (Orrantia 2004).

En paralelo, se desarrolló un índice denominado Interés de Conservación, en el que se tuvieron en cuenta parámetros definidos por las funciones descritas para el bosque natural (Loidi 1994):

$\mathrm{IC}=(\mathrm{N}+\mathrm{P}+\mathrm{A}+\mathrm{F}+\mathrm{R}) \times \mathrm{RC} \times \mathrm{S} \times \mathrm{Ag} \times \mathrm{E}$

Así, la suma de valores de $\mathrm{N}+\mathrm{P}+\mathrm{A}+\mathrm{F}$ $+\mathrm{R}$ denominada valor biológico $(\mathrm{B})$ por Loidi (1994), se inscribe en una formulación mas amplia que incluye 4 factores que dimensionan la contribución de las unidades de paisaje en términos de retención de carbono, protección de suelo y calidad de agua y necesidad territorial

$$
\mathrm{IC}=\mathrm{B} \times \mathrm{RC} \times \mathrm{S} \times \mathrm{Ag} \times \mathrm{E}
$$

El significado de los términos (parámetros), así como los criterios de valoración son los descritos a continuación de forma resumida y aparecen detallados en Loidi et al., (2007).

$\mathbf{N}=$ Naturalidad: 0-10. Entendido como el grado de influencia humana en UP; distancia a la clímax

$\mathbf{P}=$ Reemplazabilidad: $0-10$. Estima de la capacidad para recuperarse tras una perturbación

A = Amenaza: 0-10. En función de las circunstancias socioeconómicas. 
CUADRO 1

Unidades de paisaje presentes en el estudio y su código, cuenca del río Golako, España

TABLE 1

Landscape Units in the study site and its code, Golako river basin, Spain

Unidad de paisaje

Aliseda

Robledal acidófilo

Robledal mesofítico/ neutrobasófilo

(Quercus robur, Q. petraea, Q. pubescens)

Hayedo (Fagus sylvatica)

Abedular (Betula celtiberica)

Robledal en fase de regresión o juvenil

Pradera, campiña, zona de pasto, lastonar

Brezal argomal

Brezal calcícola

Pinar (Pinus spp.)

Eucaliptal (Eucalyptus spp.)

Frondosas (de hoja caduca)

Zona agrícola y rural

$\mathbf{F}=$ Valor florístico-fitocenótico: 0-10. Mide el valor biológico intrínseco: biodiversidad s.l., estructura, etc.

$\mathbf{R}=$ Rareza: $0-10$. Representa la distancia media entre los lugares donde aparece.

RC $=$ Retención de Carbono: 0-2. Valor de las Formaciones forestales en términos de depósito de carbono.

$\mathbf{S}=$ Protección del suelo: $0-2$. Coeficiente de retención del suelo por parte de las raíces; edafogénesis.

Ag = Mantenimiento o mejora de la calidad del agua: 0-2. Capacidad de purificación, servicio hidrológico de la cuenca.

$\mathbf{E}=$ Coeficiente de necesidades territoriales para la protección del ecosistema: 0-2.5. Dependiente de la densidad de población $\left(\mathrm{hab} / \mathrm{km}^{2}\right)$.

Así, el valor biológico máximo alcanzable (B) es de 50, el cual, corregido por los cuatro factores de mejora propuestos, permite un máximo teórico de 1000 unidades.
UP

$\mathrm{BR}$

$\mathrm{BM}$

Bosque ribereño

Bosque maduro

BD

Bosque degradado

PA

Prado, Pastizal

BZ

Brezal

PP

Plantación de pino

PE

Plantación de eucalipto

$\mathrm{PF}$

Plantación de frondosas

ZR

Zona Rural

\section{RESULTADOS}

Las características generales del paisaje estudiado aparecen en la Fig. 1 que muestra la ocupación total de cada unidad de paisaje estudiada en el conjunto de las estaciones. Se observa una elevada presencia de la plantación de pino PP (150 ha de 250 ha estudiadas), seguida por un grupo menos importante: PA, BD y ZR, PE y a cierta distancia PF, BM, BZ y BR. Ello a pesar del sesgo evidente en la elección de las estaciones para conseguir que todas las UP estuvieran presentes en el estudio.

Los valores del índice IC obtenidos (Fig. 2) muestran dos UP de elevado interés: BM y BR (bosque maduro y ribereño) con valores medios en torno a 800, seguidos por el bosque degrado (BD 500). Un tercer grupo, de valores entre los 50 y 100, engloban a PF, PA y BZ. Por último, aparecen las unidades de PP, PE y ZR.

En la siguiente figura (Fig. 3) se muestra la fragmentación encontrada en cada estación 


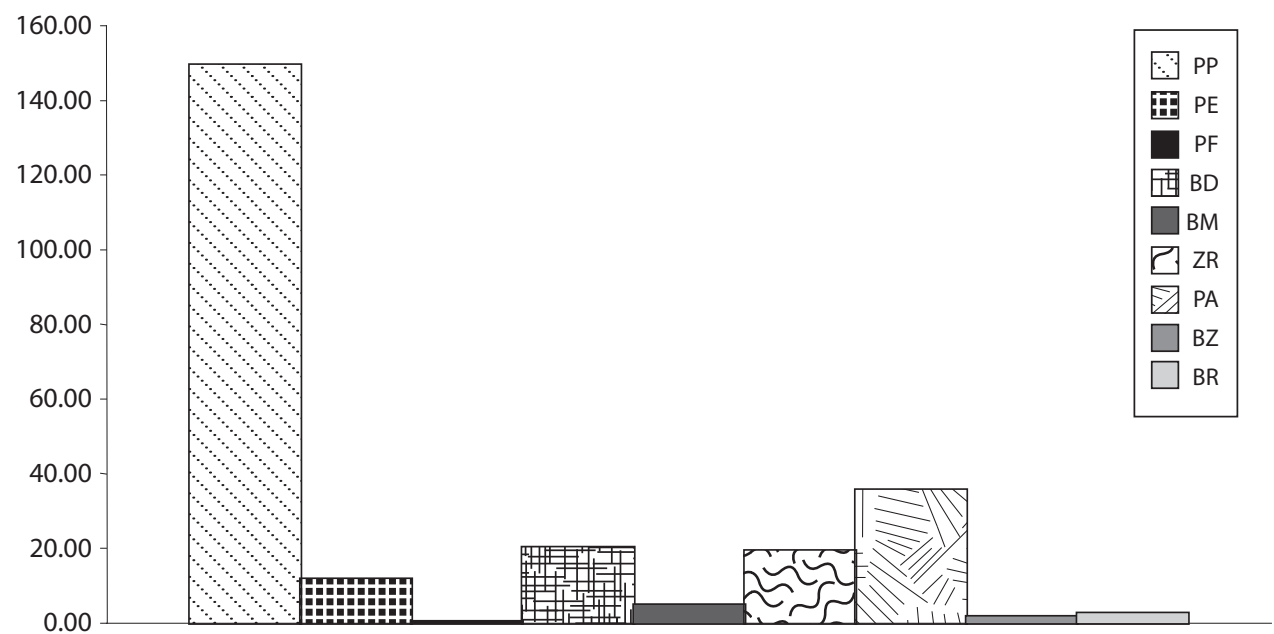

Fig. 1. Ocupación total de cada unidad de paisaje (UP) en el conjunto de las estaciones (Ha / UP), cuenca del río Golako, España. PP, plantación de pino; PE, plantación de eucalipto; PF, plantación de frondosas; BD, bosque degradado; BM, bosque maduro; ZR, zona rural; PA, pastizales; BZ, brezal argomal; BR, bosque ribereño. Fuente: Orrantia 2004.

Fig. 1. Total extension for each landscape unit (LU) in all the stations (Ha/ LU), Golako river basin, Spain. PP, pine plantation; PE, eucalyptus plantation; PF, broad leaf tree plantation; BD, degraded forest; BM, mature forest; ZR, rural area; PA, pasture; BZ, heather; BR, riparian forest. Source: Orrantia 2004.

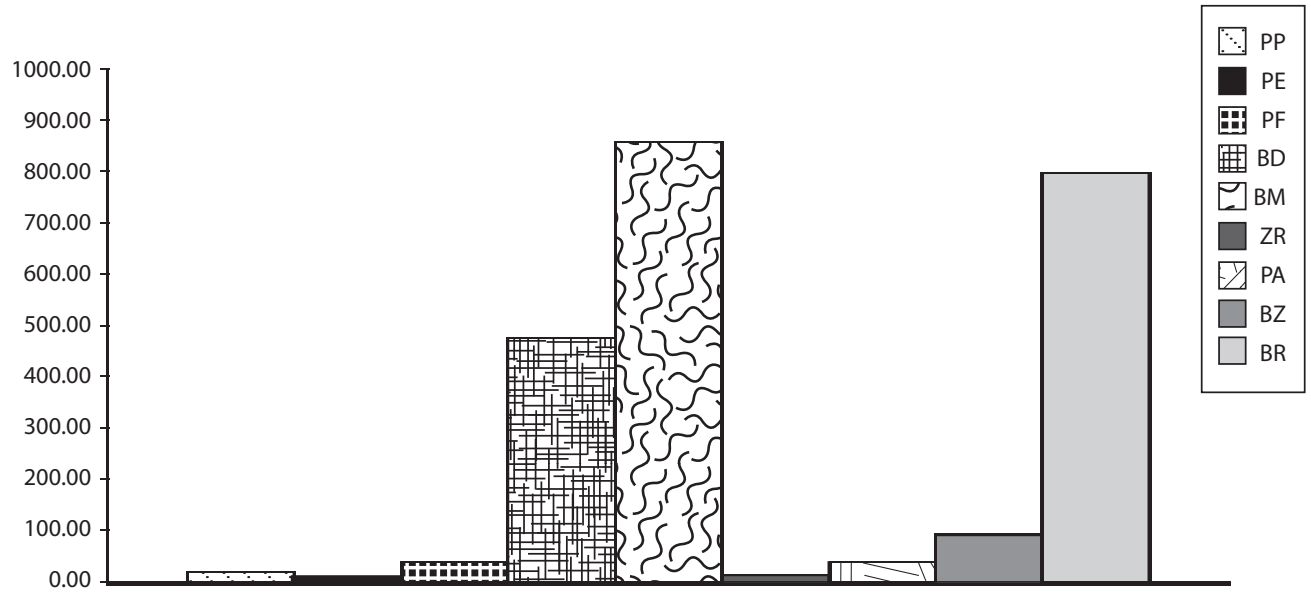

Fig. 2. Índice de interés de conservación (IC) por hectárea estudiado para cada unidad de paisaje (UP), cuenca del río Golako, España. PP, plantación de pino; PE, plantación de eucalipto; PF, plantación de frondosas; BD, bosque degradado; BM, bosque maduro; ZR, zona rural; PA, pastizales; BZ, brezal argomal; BR, bosque ribereño. Fuente: Orrantia 2004.

Fig. 2. Conservation Interest Index (IC) per hectare researched for each landscape unit (UP), Golako river basin, Spain. PP, pine plantation; $\mathrm{PE}$, eucalyptus plantation; $\mathrm{PF}$, broad leaf tree plantation; $\mathrm{BD}$, degraded forest; $\mathrm{BM}$, mature forest; $\mathrm{ZR}$, rural area; PA, pasture; BZ, heather; BR, riparian forest. Source: Orrantia 2004. 


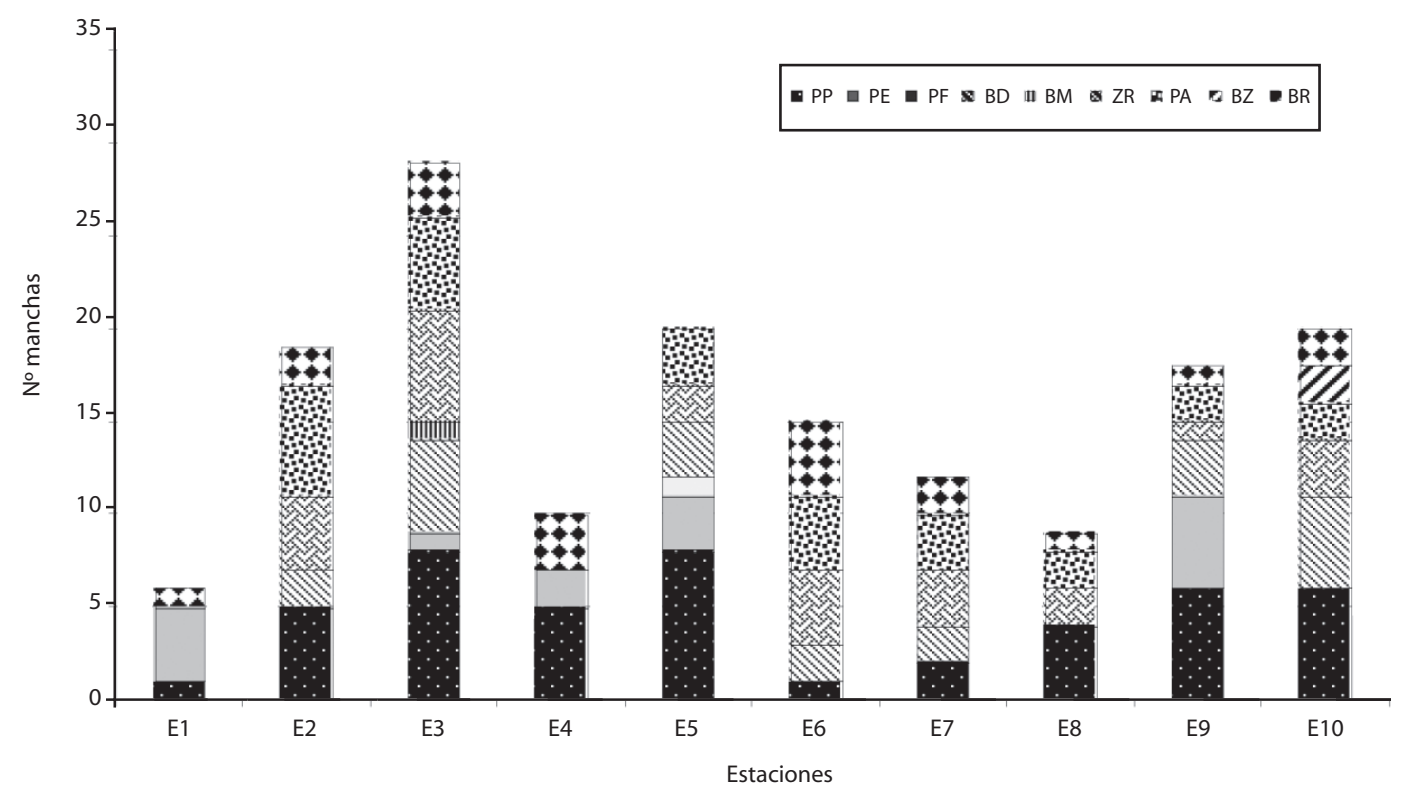

Fig. 3. Fragmentación existente por unidad de paisaje en cada estación ( $\mathrm{n}^{\mathrm{o}}$ manchas / estación), cuenca del río Golako, España. Fuente: Orrantia 2004. PP, plantación de pino; PE, plantación de eucalipto; PF, plantación de frondosas; BD, bosque degradado; BM, bosque maduro; ZR, zona rural; PA, pastizales; BZ, brezal argomal; BR, bosque ribereño.

Fig. 3. Current fragmentation per landscape unit in each plot ( $\mathrm{n}^{\mathrm{o}}$ patches / plot), Golako river basin, Spain. PP, pine plantation; PE, eucalyptus plantation; PF, broad leaf tree plantation; BD, degraded forest; BM, mature forest; ZR, rural area; PA, pasture; BZ, heather; BR, riparian forest.

y para cada unidad de paisaje: en general se observa una fragmentación del paisaje elevada. El caso más destacado es la UP Bosque Ribereño el cual, además de ocupar una superficie mínima (Fig. 1), se muestra sumamente segmentada, siendo muy amplio el fraccionamiento del bosque degradado (BD) con el $82 \%$ de las manchas estudiadas por debajo de 1 ha. En cuanto al bosque maduro, su rareza queda constatada por la presencia de una única muestra, el robledal de Arratzu, si bien de cinco hectáreas.

Así mismo, cabe destacar que se ha localizado una única parcela de PF en el área de estudio, cuando las especies caducifolias forman parte de la vegetación potencial del lugar. La utilización de especies perennes en la silvicultura causa un efecto negativo a la biodiversidad de la zona de estudio ya que las características de Eucalyptus sp. y Pinus sp. impiden el crecimiento de fauna y flora asociada a estas plantaciones. Por citar algunos casos: la falta de la ventana de luz que permitiría florecer a la flora autóctona, el desfase entre la floración (invernal en el caso del eucalipto) y la necesidad de alimento de aves reproductoras, la falta del estrato herbáceo y arbustivo, etc. consecuencias derivadas de la influencia de los aceites esenciales producidos por el eucalipto, por sus características anatómicas o por las técnicas de aprovechamiento forestal (Loidi 2005).

Estos resultados muestran que las UP cuya conservación y recuperación se considera prioritaria ocupan áreas reducidas, y en consecuencia, se ha propuesto que la fórmula de conversión de los valores de IC (calidad ecosistémica) obtenidos en primas monetarias (PSA) contemple un factor de corrección negativo para el efecto de la superficie. La ecuación se muestra a continuación: 


$$
\mathrm{PSA}=\mathrm{K} \times \mathrm{IC}_{\mathrm{ac}} /(1+\mathrm{LnS})
$$

$\mathrm{K}$ : constante de ajuste

$\mathrm{IC}_{\mathrm{ac}}$ : valor de IC acumulado de la propiedad en cuestión (IC x ha)

S: superficie en hectáreas

PSA: Pago por servicios ambientales a propietario rural

Para comprender el funcionamiento de la fórmula se realiza una simulación, en la que se toman valores de manchas obtenidos en el trabajo y se interpretan como si dichas manchas fueran propiedades. Se seleccionan 10 de diferentes tamaños, que se muestran en el Cuadro 2.

Los valores obtenidos para $\mathrm{S}$, el valor de IC acumulado ( $\left.\mathrm{IC}_{\mathrm{ac}}\right)$ y la división $\left(\mathrm{IC}_{\mathrm{ac}} /(1+\mathrm{Ln}\right.$ S)) se han trasladado a la siguiente figura (Fig. 4a) en la que se observa que, aunque el valor de IC prima las grandes extensiones de terreno con una calidad ecosistémica notable, su conversión en PSA corrige y evita que exista una relación directa entre superficie y percepción de compensación económica.

Con ello, lo que se pretende es crear una política de "primas compensatorias" para pequeños propietarios, alcanzando un objetivo doble: mantener la población rural en el campo y aumentar el número de hectáreas con bosque natural.

Las figuras siguientes (Fig 4b y 4c) muestran el funcionamiento para propiedades de una superficie entre media hectárea y diez. En el primer caso, superficies menores de una hectárea, el valor obtenido de la ecuación dobla el valor de la calidad ecosistémica, potenciando de esta manera la protección de pequeñas superficies que podrían funcionar como reservorio de hábitats para futuras actuaciones.

\section{DISCUSIÓN}

Desde esta investigación se recomienda utilizar el PSA como instrumento para favorecer la conservación de los hábitats y de las especies naturales. Tal y como apuntan los estudios consultados (FAO 2003, Grieve 2003a, Pagiola et al. 2004, Scherr et al. 2006), el uso de un índice que evalúe la calidad ecosistémica de un área permitirá ofrecer una recompensa económica en base al estado de naturalidad de la propiedad, así como hacer un seguimiento para controlar que el "servicio" por el cual se está pagando esté llevándose a cabo. Proteger la biodiversidad y la riqueza ecológica de nuestro entorno aporta beneficios cuantitativos

CUADRO 2

Simulación de funcionamiento de la conversión propuesta

TABLE 2

Operation simulation of the proposed conversion

$\begin{array}{ccccc}\text { Propietarios } & \text { Superficie (ha) } & \mathrm{IC}_{\mathrm{ac}} & 1+\ln \mathrm{S} & \mathrm{IC}_{\mathrm{ac}} /(1+\ln \mathrm{S}) \\ 1 & 0.6 & 18 & 0.49 & 36.73 \\ 2 & 1.8 & 214 & 1.59 & 134.59 \\ 3 & 2.2 & 118 & 1.79 & 65.92 \\ 4 & 9.6 & 741 & 3.26 & 227.30 \\ 5 & 16.9 & 4013 & 3.83 & 1047.78 \\ 6 & 25.5 & 10614 & 4.24 & 2503.30 \\ 7 & 132.4 & 1518 & 5.89 & 257.72 \\ 8 & 320.1 & 25142 & 6.77 & 2584.93 \\ 9 & 681.2 & 641718 & 7.52 & 3343.35 \\ 10 & 2614.5 & & 8.87 & 72347.01\end{array}$



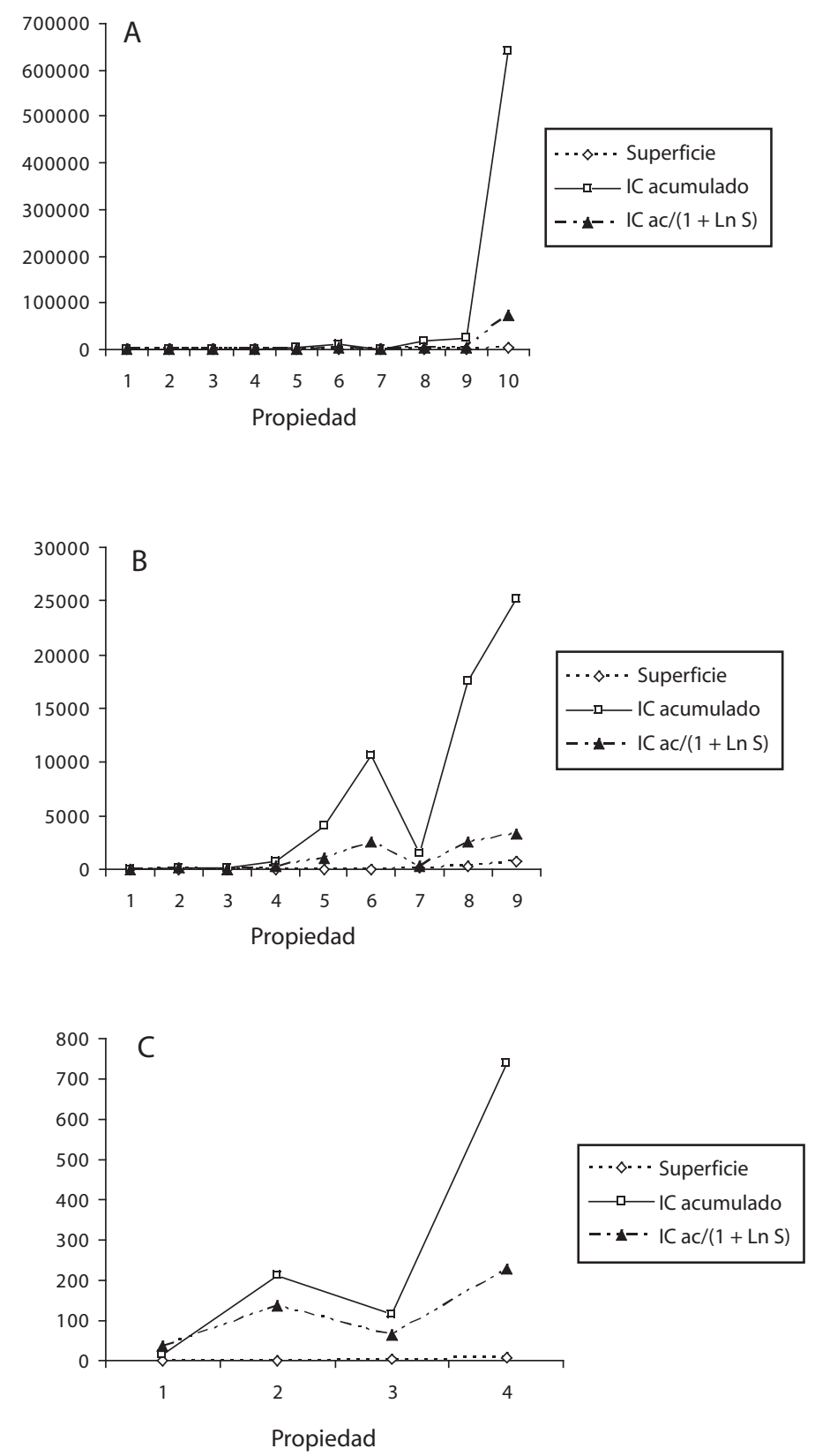

Fig. 4. Simulación del funcionamiento de la conversión propuesta. (a) Resultados para las propiedades ficticias 1 a 10. (b) Resultados logrados para las propiedades 1 a 9. (c) Resultados logrados para las propiedades 1 a 4.

Fig. 4. Operation simulation of the proposed conversion. (a) Results for the fictitious estates 1 to 10 . (b) Results obtained for states 1 to 9. (c) Results obtained for estates 1 to 4 . 
al ser humano y, por ello, el propietario cuyos terrenos tienen cierta calidad ecosistémica debe ser económicamente premiado e incentivado.

Las cifras calculadas de IC muestran que las unidades de paisaje que obtienen valores elevados realizan una función importante en el mantenimiento de la calidad ecosistémica de la cuenca, que deben ser interpretados en términos de beneficios (servicios ambientales) sobre los asentamientos humanos, no sólo del territorio en cuestión (cuenca del Golako), sino sobre una zona geográfica más amplia: la Reserva de la Biosfera de Urdaibai y su entorno geográfico.

Desde una perspectiva de conservación, la función que cumplen las áreas agrícolas y las manchas boscosas a ellas asociadas está siendo reconocida dentro de políticas de financiacion de esquemas agroambientales (AES). Áreas sujetas a dicho esquema podrían ver aumentada su capacidad para hacer frente al cambio climático, a la fragmentación del hábitat y al aumento de la presencia de especies exóticas invasoras (Donald y Evans 2006), así como actuar de reservorio de semillas, refugio de especies de interés para la polinización de los cultivos (Öckinger y Smith 2007), conservación de flora y fauna nativa, reducción de la erosión y de la contaminación difusa por pesticidas y potenciación del control de pestes por especies depredadoras naturales (Olson y Wäckers 2007). En este marco, los resultados derivados de nuestro estudio en una cuenca atlántica europea, caracterizada por la coexistencia de prácticas agrícolas tradicionales con un cierto grado de naturalidad paisajística y de la que forma parte el bosque (en distintos estados de conservación, i.e. valores de IC) mostrarían versatilidad en relación a su implementación en políticas de conservación en el contexto de los sistemas agrícolas mesoamericanos (Harvey et al. 2008).

El conjunto de trabajos realizados bajo la filosofía de AES sugieren que los valores otorgados en el presente estudio a las unidades PA y ZR deberán de incrementarse si se observan cambios con respecto a los servicios ambientales prestados, siguiendo así las directrices marcadas por las actuales políticas de conservación del territorio.

Así mismo, constatamos la necesidad de seguir desarrollando el índice para primar aquellas unidades de paisaje para las que se están observando ciertas necesidades de protección desde estamentos de la Unión Europea, tal es el caso de los brezales, además de los ya citados pastos. Así como estudiar el parámetro de Suelo del índice teniendo en cuenta las limitaciones técnicas de la metodología aplicada y las tendencias actuales (FAO 2002).

Por otro lado, es evidente que ciertas unidades no colaboran en el aumento del servicio ambiental de la cuenca, y, en el caso de las plantaciones forestales, éstas son especialmente dañinas para con la biodiversidad, el suelo, la calidad del agua, especialmente si se utilizan técnicas agresivas de silvicultura. Por ello, se considera que se debe plantear el papel que las actuales subvenciones a la plantación de pino y de eucalipto están realizando, al haberse transformado en incentivos perversos que contradicen las actuales políticas a favor del desarrollo sostenible.

El Índice IC desarrollado se fundamenta en los beneficios reconocidos por el esquema costarricense: la protección del recurso hídrico está representado por Ag, la conservación de la biodiversidad es el valor biológico (B, suma de Naturalidad, Reemplazabilidad, Amenaza, Valor Florístico-fitocenótico, Rareza), la belleza escénica evoluciona hacia el concepto del Coeficiente de necesidades territoriales (E) y la retención de carbono es RC. En IC aparece una valoración del suelo $(\mathrm{S})$ no reconocida por el sistema costarricense. Las tres unidades de paisaje valoradas inicialmente (plantaciones, bosques y sistemas agrícolas) se desarrollan en las 9 UP del estudio: BR, BM, BD, PA, BZ, PP, PE, PF, ZR. Sin embargo, en el estudio se hace una valoración in situ de estos parámetros, aspecto que hasta ahora no era desarrollado en el esquema oficial de PSA de Costa Rica (Pagiola 2002).

Las estrategias y planes de conservación atienden a las diferentes necesidades sociales y ecológicas regionales y redundan por tanto 
en una disparidad en los intereses y objetivos planteados. En países de Centro y Sudamérica (Nicaragua, Costa Rica, Colombia) existen iniciativas privadas que desarrollan un pago por servicios ambientales por la conservación de la biodiversidad y secuestro de carbono en paisajes agrícolas en base al incremento de los servicios ofrecidos por el propietario (Pagiola et al. 2004). El índice del servicio ambiental desarrollado es igual a la suma de dos índices, el de biodiversidad y el de secuestro de carbono, los cuales varían dependiendo exclusivamente del uso de la tierra. Si bien existe una limitación de base científica en este planteamiento, facilita el uso de un índice por personal no cualificado. Análogamente, se han realizado trabajos en Nueva Gales del Sur (Australia) en los que los objetivos de secuestro de carbono, mitigación de la salinidad, mitigación de suelos sulfatados, promoción de la biodiversidad, retención de suelo y mejora de la calidad de agua se sitúan en el contexto de un nuevo mercado económico creado para promover el cambio de uso de la tierra en propiedades privadas (Grieve 2003 a y b, Montagu et al. 2003, Oliver y Parkes 2003).

En cualquier caso, subyace una unidad de base dentro de las distintas formulaciones de PSA en lo que respecta a la necesidad perentoria de diseñar políticas de conservación eficaces. Es por ello que consideramos que se deben favorecer estudios de intercalibrado entre los distintos índices que permitan el desarrollo sobre bases científicas de metodologías comunes, preferentemente sencillas y fácilmente trasladables a los diferentes actores y ecosistemas. Dichos métodos incluyen dos capítulos diferenciados: a) las herramientas científico-técnicas (diseño e implementación de los índices de valoración ecosistémica) y b) las herramientas de gestión (económica, administrativa y política del PSA). En este segundo apartado la experiencia pionera de Costa Rica proporciona elementos que permiten ahorrar etapas a países que se incorporan a prácticas de conservacion del territorio.

Con este trabajo queremos contribuir al desarrollo de líneas de actuación (metodologías para otorgar primas económicas) basadas en índices de conservación del territorio, de aplicación interregional.

\section{AGRADECIMIENTOS}

Agradecemos a todas aquellas personas que han contribuido de manera directa o indirecta al desarrollo de esta investigación y su posterior publicación. Este trabajo ha sido sufragado en parte por el programa ALFAGIACT de la Unión Europea y es el resultado de la investigación de Oreina Orrantia para la obtención del título de Magister Scientiae de la Maestría en gestión integrada en áreas costeras tropicales (UCR, Costa Rica, 2002-2004).

\section{RESUMEN}

Se presenta la síntesis de un estudio piloto llevado a cabo en la Reserva de la biosfera de Urdaibai (España atlántica), en la cuenca del río Golako, donde se estudió 250 hectáreas durante 8 meses. El objetivo fue definir un índice cuantitativo de conservación del territorio (IC), basado en la evaluación individual de 9 unidades de paisaje (UP) que sintetizan las características de la zona. Luego, se formuló un método de compensación económica (PSA = euros), que permite la conservación de las áreas naturales de calidad que son propiedad privada. Dicho planteamiento se basa en la premisa de que el bosque natural, como todos los ecosistemas naturales, posee un valor social que puede ser cuantificado. Los resultados del estudio indican que el $60 \%$ del territorio está representado por plantaciones de Pinus radiata, y que la superficie ocupada por el bosque natural en estados variables de conservación, está extraordinariamente fragmentada ( $82 \%$ por debajo de 1 ha). En este contexto, se desarrolla un concepto de Pago por Servicios Ambientales (PSA) propio, diseñado tomando en cuenta: las características específicas del territorio; los estudios de Costa Rica; los planes estratégicos y jurídicos vascos; el estudio del impacto de la actividad forestal; y las funciones del bosque natural como estabilizador de sistema.

Palabras clave: servicios ambientales, valoración cuantitativa de la vegetación, índice de interés para la conservación, país Vasco, Reserva de la biosfera de Urdaibai.

\section{REFERENCIAS}

Campbell, I.C. \& T.J. Doeg. 1989. Impact of timber harvesting and production on streams: a review. Aust. J. Mar. Freshwater Res. 40: 519-39.

Donald, P.F. \& A.D. Evans. 2006. Habitat connectivity and matrix restoration: the wider implications of agrienvironmental schemes. J. App. Ecol. 43: 209-218. 
Edeso, J.M., A. Merino, M.J. González \& P. Maruri. 1999. Soil erosion under different harvesting managements in steep forestlands from northern Spain. Land Degradation \& Development. 10: 79-88.

Elosegi, A., X. Arana, A. Basaguren \& J. Pozo. 1995. Environmental auditing, self-purification processes along a médium-sized stream. Environmental Management. 19: 931-939.

FAO 2002. Relaciones tierra-agua en cuencas hidrográficas rurales. Boletín de Tierras y Aguas de la FAO. FAO, Roma, Italia.

FAO 2003. Sistemas de pago por servicios ambientales en cuencas hidrográficas. Foro Regional. Land and Water Discussion Paper 3. FAO, Roma, Italia.

FONAFIFO 2003. El desarrollo del sistema de pago por servicios ambientales en Costa Rica. FONAFIFO, San José, Costa Rica.

Gobierno Vasco 1990. Mapa de Vegetación de la Comunidad Autónoma del País Vasco. 1: 25.000 Hoja 38. Viceconsejería de Medio Ambiente, Vitoria/ Gasteiz, País Vasco, España.

Gobierno Vasco 2002. Estrategia Ambiental Vasca de Desarrollo Sostenible, EADS (2002-2020). Ihobe, Bilbao, País Vasco, España.

Grieve, A. 2003 a. An overview of the environmental service indices developed for use in the NSW Environmental Services Scheme. State Forest of New South Wales, Nueva Gales del Sur, Australia.

Grieve, A. 2003 b. Developing new income streams for farmers. NSW Environmental Services Scheme. State Forest of New South Wales, Nueva Gales del Sur, Australia.

Harvey, C.A., O. Komar, R. Chazdon, B.G. Ferguson, B. Finegan, D.M. Griffith, M. Martínez-Ramos, H. Morales, R. Nigh, L. Soto-Pinto, M. van Breugel \& M. Wishnie. 2008. Integrating agricultural landscapes with biodiversity conservation in the Mesoamerican hotspot. Conservation Biology. 1, 8-15.

Loidi, J. 1994. Phytosociology applied to nature conservation and land management, p. 17-30. In: Song, Y., Dierschke, H. \& Wang, X. (eds.). Proceed. 36th IAVS Symp. in Shanghai. East China Normal Univ. Press, Shanghai, China.

Loidi, J., I. Biurrun \& M. Herrera. 1997. La vegetación del centro-septentrional de España. Itinera Geobot. 9: $161-618$
Loidi, J. 2005. Bizkaiko basoak/ Los bosques de Bizkaia. Diputación Foral de Bizkaia, Bilbao, País Vasco, España.

Loidi ,J., M.M.Ortega \& O.Orrantia. 2007. Vegetation science and the implementation of the Habitat Directive in Spain: up-to-now experiences and further development to provide tools for management. Fitosociologia, 44 (suppl.1): 9-16.

Maridet, L., H. Piégay, O. Gilard \& A. Thévenet. 1996. L'embâcle de bois en rivière: un bienfait écologique? Un facteur de risques naturels? La Houille Blanche. 5: $32-38$.

Meaza, G. \& J.A. Cadiñanos. 2000. Valoración de la vegetación, p. 195-238. In: G. Meaza (ed.). Metodología y práctica de la Biogeografía. Serbal, Barcelona, Cataluña, España.

Montagu, K.D., A. Cowie, A. Rawson, B.R. Wilson \& B.H. George. 2003. Carbon sequestration predictor for land use change in inland areas of New South Wales Background, user notes, assumptions and preliminary model testing, Version 2.0. Tech. Pap. 68. Res. Dev. Div., State Forest, Nueva Gales del Sur, Australia.

Montori, A.; G. Llorente, M. Carretero \& X. Santos. 2001. La gestión forestal en relación con la herpetofauna, p. 252-289. In: Conservación de la biodiversidad y gestión forestal, su aplicación en la fauna vertebrada. Camprodon i Subirachs, J., Plana Bach, Univ. Barcelona, Barcelona, Cataluña, España.

Pagiola, S. 2002. Paying for water services in Central America: learning from Costa Rica, p. 37-61. In S. Pagiola, J. Bishop and N. Landell-Mills (eds.). Selling forest environmental services: Market based mechanisms for conservation and development. Earthscan, Londres, Reino Unido.

Pagiola, S., P. Agostini, J. Gobbi, C. de Haan, M. Ibrahim, E. Murgueitio, E. Ramírez, M. Rosales, J.P. Ruíz. 2004. Paying for biodiversity conservation services in agricultural landscapes. Environment Department Paper No. 96. The World Bank, Washington, EEUU.

Öckinger, E. \& H.G. Smith. 2007. Semi-natural grasslands as population sources for pollinating insects in agricultural landscapes. J. App. Ecol. 44: 50-59.

Oliver, I. y D. Parkes. 2003. A prototype toolkit for scoring the biodiversity benefits of land use change. Version 5.1. Department of Infrastructure, Planning and Natural Resources, Nueva Gales del Sur, Australia.

Olson, D.M. y F.L. Wäckers. 2007. Management of field margins to maximize multiple ecological services. J. App. Ecol. 44: 13-21. 
Orrantia, O. 2004. El pago por servicios ambientales como herramienta para promover la conservación de recursos naturales en el País Vasco. Tesis de Master (MSc). Universidad de Costa Rica.

Ortiz Malawasi, E., L.F. Sage Mora \& C. Jorge Carvajal. 2003. Impacto del programa de Pago de Servicios Ambientales en Costa Rica como medio de reducción de la pobreza en los medios rurales. Informe Final. Unidad Regional de Asistencia Técnica (RUTA). San José, Costa Rica.

Rivas-Martínez, S., F. Fernández-González, J. Loidi, M. Lousã \& A. Penas. 2001. Syntaxonomical checklist of vascular plant communities of Spain and Portugal to association level. Itinera Geobot. 14: $5-341$.
Scherr, S.J., M.T. Bennett, M. Loughney \& K. Canby. 2006. Developing future ecosystem payments in China: Lessons learned from international experience. China Council for International Cooperation on Environment and Development (CCICED) Taskforce on Ecocompensation \& Forest Trends. Peking University, Pekin, China.

Vinicio, M. 1998. Financiamiento de bosques y plantaciones forestales: pago por servicios ambientales. Revista Forestal Centroamericana. 22: 13-20.

Yeakley, J.A., D.C. Coleman, B.L. Haines, B.D. Kloeppel, J.L. Meyer, W.T. Swank, B.W. Argo, J.M. Deal \& S.F. Taylor. 2003. Hillslope nutrient dynamics following upland riparian vegetation disturbance. Ecosystems. 6: 154-167. 